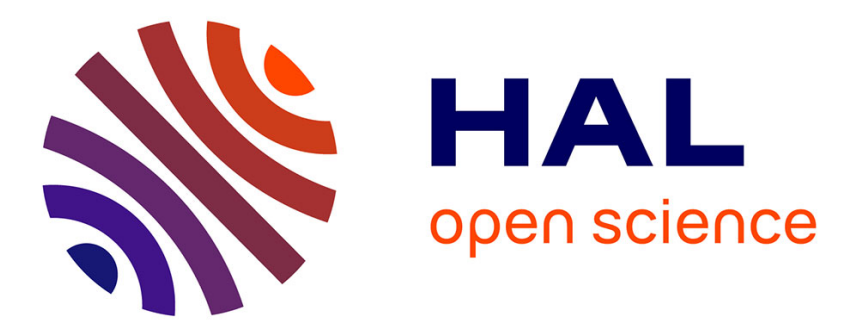

\title{
Adaptive control scheme for grid-connected photovoltaic systems with unknown bounds
}

Fernando Jaramillo-Lopez, Godpromesse Kenné, Gilney Damm, Françoise Lamnabhi-Lagarrigue

\section{- To cite this version:}

Fernando Jaramillo-Lopez, Godpromesse Kenné, Gilney Damm, Françoise Lamnabhi-Lagarrigue. Adaptive control scheme for grid-connected photovoltaic systems with unknown bounds. 11th IFAC International Workshop on Adaptation and Learning in Control and Signal Processing (ALCOSP 2013), Jul 2013, Caen, France. pp.671-676, 10.3182/20130703-3-FR-4038.00055 . hal-00880272

\section{HAL Id: hal-00880272 https://hal.science/hal-00880272}

Submitted on 30 Mar 2021

HAL is a multi-disciplinary open access archive for the deposit and dissemination of scientific research documents, whether they are published or not. The documents may come from teaching and research institutions in France or abroad, or from public or private research centers.
L'archive ouverte pluridisciplinaire HAL, est destinée au dépôt et à la diffusion de documents scientifiques de niveau recherche, publiés ou non, émanant des établissements d'enseignement et de recherche français ou étrangers, des laboratoires publics ou privés. 


\title{
Adaptive control scheme for grid-connected photovoltaic systems with unknown bounds *
}

\author{
Fernando Jaramillo-Lopez* Godpromesse Kenne** \\ Gilney Damm ${ }^{* * *}$ Francoise Lamnabhi-Lagarrigue* \\ * Laboratoire des Signaux et Systèmes, Supelec, Plateau du Moulon, \\ 91192 Gif-sur-Yvette, France (e-mail: jaramillo@lss.supelec.fr) \\ ** LAIA, Département de Genie Electrique, IUT-FV Bandjoun \\ Université de Dschang, Cameroun (e-mail: gokenne@yahoo.com) \\ *** IBISC, Université Evry, Val d'Essonne, France (e-mail: \\ gilney.damm@ibisc.fr)
}

\begin{abstract}
An adaptive control scheme for maximum power point tracking of a single-phase grid-connected photovoltaic system is presented. The difficulty on design a controller that may operate a photovoltaic system on its maximum power point (MPP) is that, this MPP depends on temperature and solar irradiance, ambient conditions that are time-varying and difficult to measure.

A solution using an on-line sliding mode estimator is presented. It estimates three different parameters that depends on solar irradiance and temperature, eliminating the necessity of having any sensor for these environmental variables. It is capable of estimate time-varying parameters. A complete analysis was done taking into account the non-linearities showed by the closed-loop system. A variation of the Lyapunov redesign technique was used to find a controller that give globally asymptotically stable trajectories of the closed-loop system.

An adaptive law was found to substitute a perturbation bound and also to eliminate possible chattering due to the discontinuous controller term.

Computer simulations are presented to show the good performance of the controller.
\end{abstract}

Keywords: Adaptive control; Parameter estimation; Global stability; Solar cells; Chattering.

\section{INTRODUCTION.}

The compromising situation of the environment due to the pollution, and the high costs of fossil fuels have originated new policies and regulations that have stimulated the interest on alternative energy sources. Developing countries have increased in an important way the penetration of these energy sources (IEA [2012]).

One of the most widely used renewable energy systems are the photovoltaic systems (PVS), which converts sunlight directly into electricity by means of a semiconductive process. PVS usually consists of a photovoltaic panel or array, a power conditioning system (PCS), and occasionally, an energy storage element. Most of the grid-connected PVS (GPVS) consist of the first two elements.

Two of the topologies used for low and medium power levels are the two-stage $\mathrm{DC} / \mathrm{DC}+\mathrm{DC} / \mathrm{AC}$, and the single-stage DC/AC converter. In Jain et al. [2007], the authors show the main advantages of the single-stage against the two-stage topology. In Kjaer et al. [2005] and Xue et al. [2004], the authors analyze advantages and disadvantages of various single-phase grid-connected

\footnotetext{
* This research has received partially support from European Union Seventh Framework Programme [FP7/2007-2013] under grant agreement no.257462 HYCON2 Network of excellence.
}

inverters topologies. The single-phase single-stage central inverter DC/AC topology was chosen in this work, because of its simplicity and low price.

The output power of a PV array is function of irradiance and temperature. So, changes in these environmental conditions will bring different power values. To increase the efficiency of the overall system, PVS always needs to work in its maximum power point (MPP), to deliver the maximum amount of energy (Fig. 1). Hence, an algorithm that can follow these power changes is needed. This is the maximum power point tracking (MPPT) algorithm. There are several MPPT algorithms like perturb and observe, incremental conductance, extremum-seeking among others. They compute the value of the voltage corresponding to the MPP. This voltage is then used as a reference value in the controller. In Femia et al. [2005] and Esram et al. [2007], the authors present some improvements and comparisons between these algorithms.

Previous works on MPPT control of PVS were done by splitting the problem in two parts: a control capable of tracking the MPP and a control capable of deliver a sinusoidal current in phase with the grid voltage - unity power factor-.

Solutions like feedback linearization and sliding mode techniques were applied to the first part. 


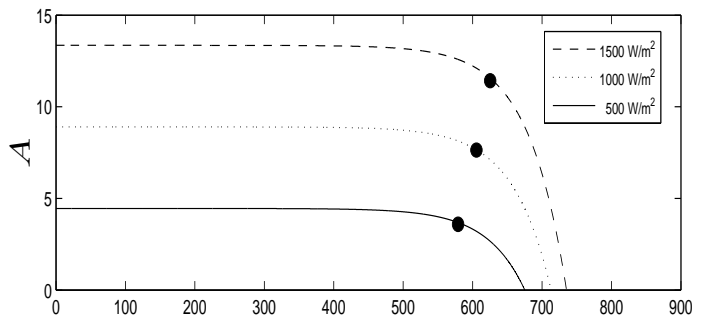

a) voltage vs current curve at different irradiance levels

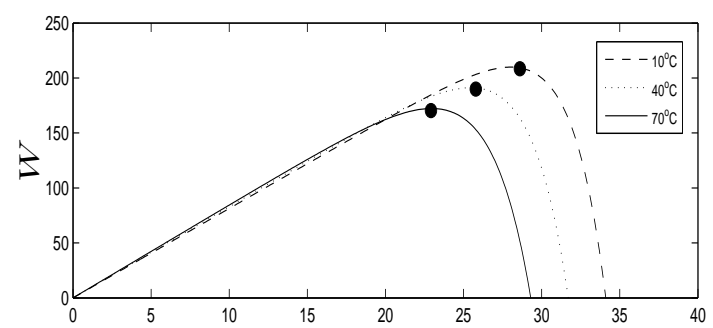

b) voltage vs power curve at different temperatures

Fig. 1. MPP of a typical PV array for different environmental conditions a) $T=25^{\circ} \mathrm{C}$ and different irradiance values. b) $G=1000 \mathrm{~W} / \mathrm{m}^{2}$ and different temp. values.

Several solutions have been developed for the second part, like the $\mathrm{P}+$ resonant controller (see Zmood et al. [2003]) where a better alternative to the PI controller is introduced, and the adaptive resonant controller (Timbus et al. [2006]) which is an improve of the proportional resonant controller. However, these solutions are linear based techniques. Approximations that doesn't take into account the nonlinear behavior of the PV array.

An interesting solution that uses the nonlinear model of PVS was presented in Meza et al. [2008]. In this work, the MPPT and the unity power factor tasks are satisfied with a single passive control, based on the work of Sanders et al. [1992]. MPPT is accomplished for time-varying environmental conditions, with the help of an estimator that compute a reference for the controller. However, global stability with the use of this estimator is not proved.

In Meza et al. [2012] an attempt to prove global stability for the whole system was made. In this case, an estimation of the parameter that depends on irradiance and temperature is used together with the Lyapunov-based controller to prove global stability. However, in that work it is assumed that another two parameters that depends on temperature are known, so, a temperature sensor and a very precise knowledge of the part of the model that depends on temperature are needed.

In El Fadil and Giri [2011] the authors solved the climatic sensorless problem. The main difference with the present work is the application: in that work they use the PVS to charge a battery. The objective of deliver a current in phase with the grid voltage is not present. Another important difference is that the analysis was made considering the time-varying parameters as constants.

In this paper, we are presenting a solution that uses an adaptive scheme control that is capable of achieving MPPT for changing environmental conditions and deliver

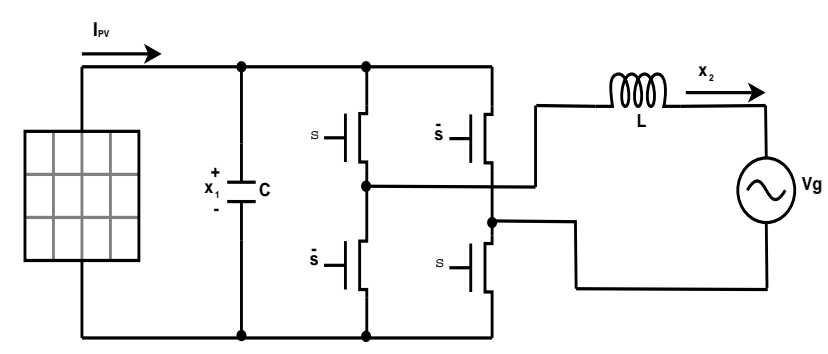

Fig. 2. Photovoltaic system analyzed in this paper.

unity power factor current to the grid. In this solution, a sliding mode estimator capable of estimate time-varying parameters was designed and it estimates parameters that depends on irradiance and temperature. No assumption about known parameters were made and global stability taking into account the nonlinear model and the estimates of the parameters was proved. The main contribution consists in the full analysis - nonlinear model, controller and estimator - of the system, increasing of the robustness and elimination of the necessity of irradiance and temperature sensors with this estimator.

\section{PROBLEM FORMULATION AND MATHEMATICAL MODEL.}

The system analyzed in this paper is shown in figure 2 and consists in a photovoltaic array of solar cells and a DC/AC PCS. The states of the system are given by the capacitor voltage $\left(x_{1}\right)$ and the inductor current $\left(x_{2}\right)$. The PCS is a full-bridge inverter driven by a bipolar PWM scheme. The PWM gives two discrete complementary signals $s$ and $\bar{s}$ which turn on and off the four switches in the PCS. The PWM block is fed by the control signal $u \in[-1,1]$.

\subsection{Mathematical model.}

The PV array is composed by PV cells arranged in series and parallel. The PV cell model used in this work is the single-diode model with no resistors. In Gow et al. [1999] and Saloux et al. [2011], the authors present some models and give explicit relations to get the electric characteristics which are functions of irradiance and temperature. In Aggarwal et al. [1997], the authors studied the PV array modeling problem for nonidentical PV cells. A comparative analysis was made in Bennett et al. [2012], and it is shown that all models have no significant differences for MPPT purposes.

The $I-V$ characteristic curve of a $\mathrm{PV}$ array with identical cells is given by:

$$
I=I_{p h}(G) N_{p}-I_{o}(T) N_{p}\left[\exp \left(\frac{q V}{n N_{s} k_{B} T}\right)-1\right]
$$

where $I_{p h}$ is the photocurrent, that depends on irradiance $G, I_{o}$ is the saturation current, that depends on temperature $T, q$ is the absolute value of electron's charge, $n$ is the quality factor of the diode, $N_{p}$ is the number of cells connected in parallel, $N_{s}$ is the number of cells connected in series, $k_{B}$ is Boltzmann's constant, $T$ is the temperature of the P-N junction and $V$ is the capacitor voltage $x_{1}$.

This equation can be written in a simple way:

$$
I\left(x_{1}\right)=c_{1}-c_{2} e^{c_{3} x_{1}}
$$


where $c_{1}$ is function of irradiance and temperature and $c_{2}$ and $c_{3}$ are functions of temperature.

The model of the whole system is given by:

$$
\dot{x}=f(t, x)+G(t, x) u
$$

where

$$
f(t, x) \triangleq\left[\begin{array}{c}
\frac{I\left(x_{1}\right)}{C} \\
\frac{-v_{g}(t)}{L}
\end{array}\right], G(t, x) \triangleq\left[\begin{array}{c}
\frac{-x_{2}}{C} \\
\frac{x_{1}}{L}
\end{array}\right]
$$

$C$ and $L$ are the known values of the capacitor and the inductor respectively, and $v_{g}$ the grid voltage. The signals that are measured are the states $x \in \mathbb{R}^{2}$, the voltage grid $v_{g} \in \mathbb{R}$ and the PV array current $I \in \mathbb{R}$, which is a common practice in this type of circuits.

$u$ is the control signal composed by the terms:

$$
u=u_{n}+w+\delta(t, x)
$$

$\delta(t, x)$ is an uncertain term that satisfies the matching condition (i.e. it enters at the same point that the control signal $u), w$ is the term that will be derived to compensate the uncertain term and $u_{n}$ is the control component that turn the nominal system globally asymptotically stable. In section IV, these components are explained in detail.

The nominal system is the system without the uncertain term $\delta(t, x)$, hence:

$$
\dot{x}=f(t, x)+G(t, x) u_{n}
$$

where $f(t, x)$ and $G(t, x)$ are defined in the same way that before.

The main tasks that the signal control $u$ must fulfill are:

(1) To track the MPP of the PV array, despite of the changes in irradiance and temperature.

(2) To deliver a current in phase with the grid voltage.

Another important issue that must be taken into account, is the total harmonic distortion (THD) injected in the grid. One way to reduce this is computing the controller references at the ending of each voltage grid cycle.

The controller needs two references to accomplish the main tasks of the system.

The first reference is the voltage $\mathrm{DC}$ value in the capacitor

$$
\bar{x}_{1 *} \in \mathbb{R}_{+}
$$

that provides the MPP and is given by a MPPT algorithm.

The second reference is the current value in the inductor $x_{2 *}$, which is taken from Meza et al. [2012]:

$$
x_{2 *} \triangleq \frac{2 v_{g}(t) \bar{x}_{1 *} I_{*}\left(\bar{x}_{1 *}, c_{i}\right)}{A^{2}}
$$

and is the inductor current value that achieves MPPT, and that has to be in phase with the grid voltage. $I_{*}$ is the PV current (2) evaluated at the voltage $\bar{x}_{1 *}, v_{g}$ is assumed to be sinusoidal with constant amplitude $A$ and frequency $\omega$, $v_{g}=A \sin (\omega t)$.

However, the parameters $c_{i}$ in $I_{*}$ are unknown. Therefore, an estimator was designed and it is presented here. It allows to calculate the complete $I_{*}$ expression.

Hence, the second reference becomes:

$$
\hat{x}_{2 *} \triangleq \frac{2 v_{g}(t) \bar{x}_{1 *} \hat{I}_{*}\left(\bar{x}_{1 *}, \hat{c}_{i}\right)}{A^{2}}
$$

where $(\hat{\cdot)}$ indicate the estimate of $(\cdot)$.

\section{ESTIMATOR DESIGN.}

\subsection{Reparametrization.}

A reparametrization was made in order to be able to design the next estimator.

From (2) and (6) the derivative of the current $I$ is:

$$
\dot{I}=-c_{2} c_{3} \dot{x_{1}} e^{c_{3} x_{1}}=\frac{1}{C}\left(u x_{2}-I\right)\left(\theta_{1}-\theta_{2} I\right)=\Phi^{T} \theta
$$

where:

$$
\theta \triangleq\left[\begin{array}{c}
c_{1} c_{3} \\
c_{3}
\end{array}\right], \Phi^{T} \triangleq \frac{1}{C}\left[u x_{2}-I,-u x_{2} I+I^{2}\right]
$$

also consider the following estimator and observer errors respectively $\tilde{\theta}=\hat{\theta}-\theta, \tilde{I}=\hat{I}-I$.

\subsection{Sliding mode estimator.}

In Kenne et al. [2008] a new framework for nonlinear systems time-varying parameter estimation using sliding mode techniques was proposed.

The following notation is used in this subsection:

$$
\left|x^{T}\right|_{G}=\left(\left|x_{1}\right|,\left|x_{2}\right|, \ldots\left|x_{n}\right|\right)
$$

$\operatorname{diag}(A)$ is the column vector whose elements are the diagonal elements of a given square diagonal matrix $A$.

The function $\operatorname{sign}(\cdot): \mathbb{R}^{n} \rightarrow \mathbb{R}^{n}$ is defined as

$$
\operatorname{sign}\left(x^{T}\right)=\left(\operatorname{sign}\left(x_{1}\right), \operatorname{sign}\left(x_{2}\right), \ldots \operatorname{sign}\left(x_{n}\right)\right)
$$

with

and

$$
x^{T}=\left(x_{1}, x_{2}, \ldots x_{n}\right)
$$

$$
\operatorname{sign}\left(x_{i}\right)=\left\{\begin{array}{lll}
-1 & \text { for } & x_{i}<0 \\
{[-1,1]} & \text { for } & x_{i}=0 \\
1 & \text { for } & x_{i}>0
\end{array}\right.
$$

Now, let us assume that

Assumption 1. The derivatives of the parameters vector $\theta$ are bounded and these bounds are known:

$$
\dot{\theta}_{i}(t) \leq \mu_{\theta_{i}}
$$

where $\mu_{\theta i}$ are known positive numbers and $i=1,2$.

In order to design the time-varying parameter estimator consider the following adaptive observer for the current $I$ :

$$
\dot{\hat{I}}=v_{I}+\Phi^{T} \hat{\theta}
$$

where

thus

$$
v_{I}=-K_{I} \operatorname{sign}(\hat{I}-I)
$$

$$
\dot{\tilde{I}}=v_{I}+\Phi^{T} \tilde{\theta}
$$

For $K_{I}$ sufficiently chosen large

$$
K_{I}>\left|\Phi^{T} \tilde{\theta}\right|
$$

and assuming that $\hat{\theta}$ is bounded (the proof will be shown later) then a sliding mode regime occurs on the manifold $\tilde{I}=0$ and $0=v_{I e q}+\Phi^{T} \tilde{\theta}$ then

$$
v_{\text {Ieq }}=-\Phi^{T} \tilde{\theta}
$$


The following approximation is used (see the work of Utkin [1992])

$$
v_{I e q}=\frac{1}{1+\tau s} v_{I}
$$

where $s$ is the Laplace operator and $\tau \rightarrow 0$ is a positive constant.

Hence

and finally

$$
\tilde{\theta}=-\Phi\left(\Phi^{T} \Phi\right)^{-1} v_{I e q}
$$

$$
\dot{\hat{\theta}}=-K_{\theta} \operatorname{sign}(\tilde{\theta})
$$

Choosing the gain matrix for the estimator such that:

$$
\operatorname{diag}\left(K_{\theta}\right)_{i}>\mu_{\theta_{i}}
$$

then, the estimated parameters $\hat{\theta}$ will converge to their real values $\theta(t)$ in finite time.

Proof. Let us consider the following Lyapunov candidate function

$$
\begin{aligned}
V & =\frac{1}{2} \tilde{\theta}^{T} \tilde{\theta} \triangleq \beta(\tilde{\theta}) \\
\dot{V} & =\tilde{\theta}^{T} \dot{\tilde{\theta}}=\tilde{\theta}^{T}\left(-K_{\theta} \operatorname{sign}(\tilde{\theta})-\dot{\theta}\right) \\
& \leq-\left|\tilde{\theta}^{T}\right|_{G}\left(\operatorname{diag}\left(K_{\theta}\right)-\mu_{\theta}\right)
\end{aligned}
$$

From (19), one can see that $\dot{V}$ is negative-definite for $\tilde{\theta} \neq 0$. Therefore, the estimation error $\tilde{\theta}$ will converge to 0 in finite time.

\section{CONTROLLER DESIGN.}

In order to accomplish the control objectives, the problem has been divided in two parts:

(1) To find the control portion that renders the nominal system (6) globally asymptotically stable.

(2) To find the control portion that compensates the uncertain term $\delta(t, x)$ and gives the whole system (3) globally asymptotically stable.

\subsection{Controlling the nominal system.}

In this part of the analysis it is considered that the $x_{2 *}$ reference is well known, i.e. the observer error is zero $\tilde{I}(t)=0$. The term $\delta(t, x)$ contains the perturbations due to $\tilde{I}(t) \neq 0$ and the second portion of the control handle it.

According to the idea given in Sanders et al. [1992], the control is decomposed in two parts: one for the steadystate stage $u_{* n}$ and another for the dynamic stage $e_{u n}$.

The reference system is:

$$
\dot{x}_{*}=f\left(t, x_{*}\right)+G\left(t, x_{*}\right) u_{* n}
$$

Hence, the control component for the steady state is:

$$
u_{* n}=\frac{L \dot{x}_{2 *}+v_{g}(t)}{x_{1 *}}
$$

Consider the following tracking errors and control variable respectively:

$$
e=x-x_{*}, e_{u n}=u_{n}-u_{* n}
$$

$\dot{e}=\dot{x}-\dot{x}_{*}=\left[\begin{array}{c}\frac{I\left(x_{1}\right)-I_{*}}{C} \\ 0\end{array}\right]+\left[\begin{array}{c}-\frac{e_{2} u_{* n}}{C}-\frac{\left(e_{2}+x_{2 *}\right) e_{u n}}{C} \\ \frac{e_{1} u_{* n}}{L}+\frac{\left(e_{1}+x_{1 *}\right) e_{u n}}{L}\end{array}\right]$

Let us propose the Lyapunov candidate function

$$
\begin{aligned}
V_{n}= & \frac{C}{2} e_{1}^{2}+\frac{L}{2} e_{2}^{2} \\
\dot{V}_{n}= & C e_{1} \dot{e}_{1}+L e_{2} \dot{e}_{2} \\
= & e_{1}\left(I-I_{*}\right)-e_{1}\left[e_{2} u_{* n}+\left(e_{2}+x_{2 *}\right) e_{u n}\right] \\
& +e_{2}\left[e_{1} u_{* n}+\left(e_{1}+x_{1 *}\right) e_{u n}\right] \\
= & -c_{2} e_{1}\left(e^{c_{3} x_{1}}-e^{c_{3} x_{1 *}}\right)-e_{u n}\left(e_{1} x_{2 *}-e_{2} x_{1 *}\right)
\end{aligned}
$$

and choose the controller for the error dynamics of the nominal system:

$$
e_{u n} \triangleq e_{1} x_{2 *}-e_{2} x_{1 *}
$$

then

$$
\dot{V}_{n}=-c_{2} e_{1}\left(e^{c_{3} x_{1}}-e^{c_{3} x_{1 *}}\right)-\left(e_{1} x_{2 *}-e_{2} x_{1 *}\right)^{2}
$$

From the fact that the first term of the right side of (25) is $<0 \forall e_{1} \neq 0$, the nominal system is globally asymptotically stable (GAS). Thus, the control component $e_{u n}$ will drive the error dynamics $e$ asymptotically to 0 .

\subsection{Controlling the whole system.}

The control part of the nominal system was established in (23) and (24). These equations are functions of $\dot{x}_{2 *}$ and $x_{2 *}$, and these values are not exactly known. Therefore, the control for the whole system is

$$
\begin{aligned}
u & =u_{* n}\left(x_{2 *}\right)+e_{u n}\left(x_{2 *}\right)+\Delta u_{*}+\Delta e_{u} \\
& =u_{* n}\left(x_{2 *}\right)+e_{u n}\left(x_{2 *}\right)+\delta(t, x)
\end{aligned}
$$

where

$$
\delta(t, x) \triangleq \Delta u_{*}+\Delta e_{u}=\frac{L \Delta \dot{x}_{2 *}}{x_{1 *}}+e_{1} \Delta x_{2 *}
$$

is the uncertain term, and $\Delta \dot{x}_{2 *}, \Delta x_{2 *}$ are the perturbations due to the transients in the observer, i.e. $\tilde{I} \neq 0$.

In order to find the controller for the whole system the next assumption is needed.

Assumption 2. The uncertain term is bounded, i.e.

$$
\|\delta(t, x)\|_{2}<\xi
$$

where $\xi$ is a unknown positive number.

Since this uncertain term satisfies the matching condition, a variation of the Lyapunov redesign technique (Khalil [1996]) is used here to compensate it.

Now, a term $w$ will be added to the controller to compensate the uncertain term:

where $u_{n}=u_{* n}+e_{u n}$

$$
u=u_{n}+\delta(t, x)+w
$$

The closed loop system becomes

$$
\dot{x}=f(t, x)+G(t, x)\left[u_{* n}+e_{u n}\right]+G(t, x)[w+\delta(t, x)]
$$

and

$\dot{e}=f(t, x)+G(t, x)\left[u_{* n}+e_{u n}\right]-\dot{x}_{*}+G(t, x)[w+\delta(t, x)]$ 
Consider the following Lyapunov candidate function for the whole system:

$$
V=\frac{C}{2} e_{1}^{2}+\frac{L}{2} e_{2}^{2}+\beta(\tilde{\theta})
$$

where $\beta(\tilde{\theta})$ was defined in (20).

Let us omit the arguments of the functions for the sake of brevity. The time derivative is:

$$
\begin{aligned}
\dot{V} & =\frac{\partial V}{\partial e}\left\{f+G\left(u_{* n}+e_{u n}\right)-\dot{x}_{*}\right\}+\frac{\partial V}{\partial e}\{G(w+\delta)\}+\dot{\beta} \\
& =\dot{V}_{n}+\dot{\beta}+\frac{\partial V}{\partial e} G w+\frac{\partial V}{\partial e} G \delta
\end{aligned}
$$

To compensate the perturbation, the following term is considered:

with the adaptive law:

$$
w \triangleq-\hat{\xi} \frac{\frac{\partial V}{\partial e} G}{\left\|\frac{\partial V}{\partial e} G\right\|_{2}}
$$

$$
\dot{\hat{\xi}}=\left\{\begin{array}{lll}
-k_{\xi} \hat{\xi} & \text { for } & |\tilde{I}| \leq \epsilon \\
\alpha & \text { for } & |\tilde{I}|>\epsilon
\end{array}\right.
$$

where $k_{\xi}, \alpha>0$ and $\epsilon \geq 0$.

First, let us analyze the system setting $\epsilon=0$. Then, when $\tilde{I}>0, \hat{\xi}(t)=\alpha t+\hat{\xi}(0)$, the perturbation term $\delta$ will be bounded and $>0$ and the next inequality will hold in finite time:

$$
\hat{\xi}\left\|\frac{\partial V}{\partial e} G\right\|_{2}>\frac{\partial V}{\partial e} G \delta
$$

Thus, from (21), (25), (31), (32) and (34), $\dot{V}$ will be negative definite and the whole system will be globally asymptotically stable (GAS) with the controller given by $u_{n}+w$. Now, when $\tilde{I}=0$ the perturbation term $\delta$ will be zero also, and the adaptive term $\hat{\xi}$ will converge to zero, "turning off" in this way, the discontinuous term $w$, and the whole system will be GAS.

In practice, the observer error $\tilde{I}$ can't be exactly zero for a large time, even when the system variations are small. This is why a small interval $\epsilon$ needs to be setting. Then, when $|\tilde{I}| \leq \epsilon$, the adaptive term $\hat{\xi}$ will converge to zero and the whole system will continue being GAS due that the perturbation term $\delta$ will be small enough that will be dominated by the negative terms in (21) and (25). In this way, we have GAS for the whole system and chattering due to the discontinuous term $w$ can be avoided.

\section{SIMULATION RESULTS.}

Numerical simulations were made in the Simulink/Matlab platform to verify the performance of the adaptive controller. The simulation consists in step changes in the irradiance and temperature variables. Its values were changed by $50 \%$. This is one of the worst conditions for MPPT in PVS. The initial conditions in the plant and in the adaptive term $\hat{\xi}$ were zero and in the estimator was $1 \times 10^{-5}$. The MPPT algorithm used in these simulations was the extremum seeking algorithm (ESA). The initial condition in this algorithm has to be set $>0$ to avoid singularities in (23). Figures 3 and 4 show the results.

The step changes in irradiance and temperature are shown in graphs a) and b) in figure 3 .
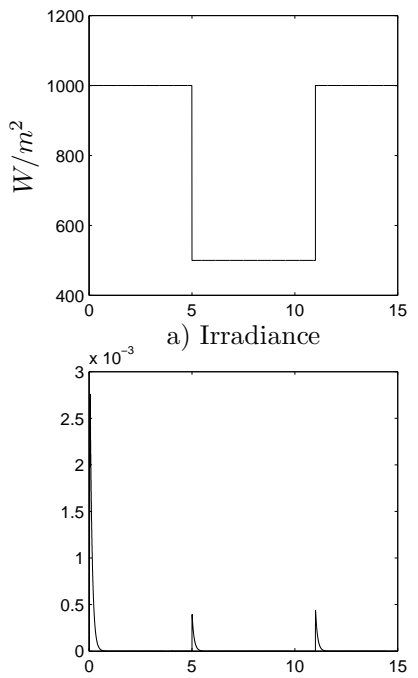

c) Adaptive bound $\hat{\xi}$

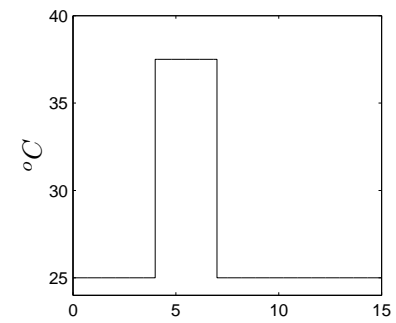

b) Temperature

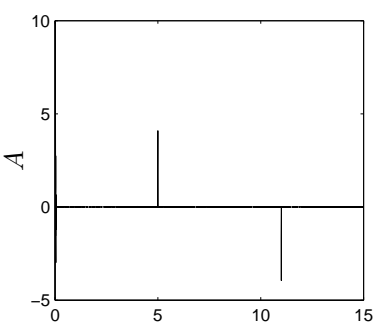

d) Observed $\tilde{I}$
Fig. 3. a) Irradiance signal b) Temperature signal c) Adaptive bound $\hat{\xi}$ d) Observer error
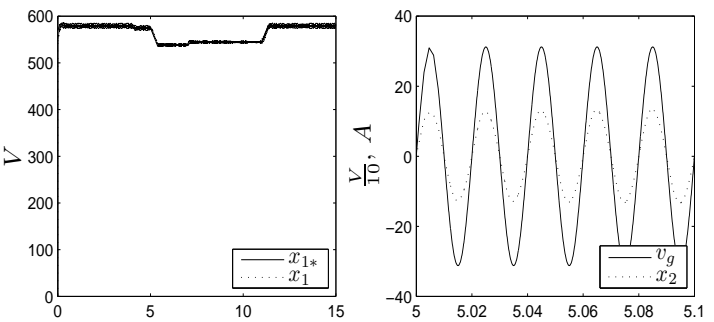

a) Capacitor voltage
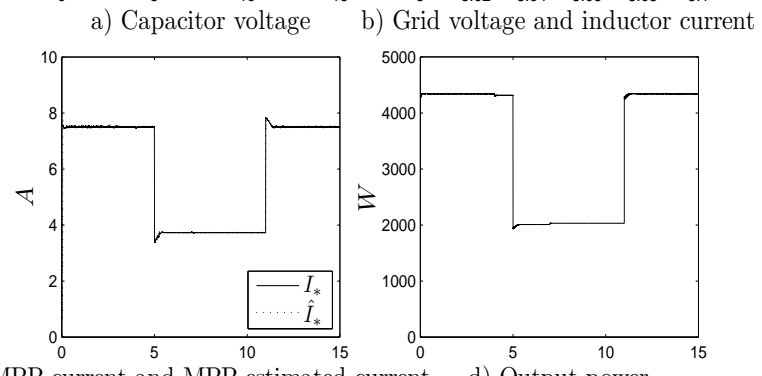

c) MPP current and MPP estimated current d) Output power

Fig. 4. a) $x_{1 *}$ and $x_{1}$ signals b) $v_{g}$ and $x_{2}$ signals c) $I_{*}$ and $\hat{I}_{*}$ signals d) Output power

The graph a) in figure 4 shows the first reference $x_{1 *}$, given by the ESA, and the capacitor voltage $x_{1}$. Both signals are almost indistinguishable. In the graph b) of the same figure we can see that the voltage grid and the injected current $\left(x_{2}\right)$ are in phase. These graphs show that the controller fulfill both objectives: MPPT of PV array and unity output power factor, under these demanding conditions.

The maximum power point current $I_{*}$ and the maximum power point current estimated $\hat{I}_{*}$ are shown in graph c) in figure 4. As we can see, the signals are almost identical, showing the good performance of the sliding mode estimator.

Graph d) in figure 4 shows the output power. This is the maximum power that the PVS can deliver with the irradiance and temperature given. It clearly follows the environmental variations. At time 4 and $7 \mathrm{sec}$. we can 
see small variations due to the stepping changes in the temperature.

In graphs c) and d) in figure 3 , the adaptive bound $\hat{\xi}$ and the observer error $\tilde{I}$ are shown. The adaptive bound starts growing when the observer error is bigger than the $\epsilon$ interval. These perturbations occur at time 5 and 11 sec. due to the irradiance variations. The perturbations due to the temperature variations are so small that they fall into the $\epsilon$ interval. Once the observer error fall into the $\epsilon$ interval, the adaptive bound $\hat{\xi}$ decrease exponentially to zero. The discontinuous controller term $w$ is different from zero only when medium and big perturbations occur, and it will be zero again as soon as the perturbations cease. In this way, the chattering problem is avoided.

\section{CONCLUSION.}

An adaptive control scheme for maximum power point tracking of a single-phase grid-connected PVS was presented. The PVS driven by this control law is capable of deliver the maximum power available under variable environmental conditions.

An estimator was designed using sliding mode techniques. It is capable of estimate time-varying parameters that depends on irradiance and temperature.

A Lyapunov function that proves globally asymptotically stability of the system was found. The analysis includes the non-linear model of the system, the dynamics of the estimator and the uncertainty in the second reference $x_{2 *}$.

An adaptive law was proposed to substitute the perturbation bound. This adaptive law helps the system to be GAS under perturbations and it is zero when the perturbations cease. The discontinuous controller term $w$ is driven by this adaptive law and the chattering problem is avoided in this manner.

Numerical simulations were made to verify the performance of the solution. It was showed the good performance of the system even under very demanding conditions.

\section{Acknowledgements}

The work of F. Jaramillo has been supported by CONACyT, Mexico and the French Embassy in Mexico.

\section{REFERENCES}

IEA PVPS PROGRAMME Task 1, "Trends in Photovoltaic Applications", IEA International Energy Agency, Survey report of selected IEA countries between 1992 and 2011, Aug. 2012

S. Jain and V. Agarwal, "A Single-Stage Grid Connected Inverter Topology for Solar PV Systems With Maximum Power Point Tracking", IEEE Trans. Power Elect., Vol. 22, No. 5, pp. 1928-1940, Sep. 2007,

S. B. Kjaer, J. K. Pedersen and F. Blaabjerg, "A Review of Single-Phase Grid-Connected Inverters for Photovoltaic Modules", IEEE Trans. Ind. Appl., Vol. 41, No. 5, pp. 1292-1306, Sep.-Oct. 2005,

Y. Xue, L. Chang, S. B. Kjaer, J. B. and T. Shimizu, "Topologies of Single-Phase Inverters for Small Distributed Power Generators: An Overview", IEEE Trans. Power Elect., Vol. 19, No. 5, pp. 1305-1314, Sep. 2004,
N. Femia, G. Petrone, G. Spagnuolo and M. Vitelli, "Optimization of Perturb and Observe Maximum Power Point Tracking Method", IEEE Trans. Power Elect., Vol. 20, No. 4, pp. 963-973, July 2005,

T. Esram and P. L. Chapman, "Comparison of photovoltaic array maximum power point tracking techniques", IEEE Trans. Energy Conv., Vol. 22, No. 2, pp. 439-449, June 2007,

D. N. Zmood and D. G. Holmes, "Stationary Frame Current Regulation of PWM Inverters With Zero SteadyState Error", IEEE Trans. Power Elect., Vol. 18, No. 3, pp. 814-822, May 2003,

A. V. Timbus, M. Ciobotaru, R. Teodorescu and F. Blaabjerg, "Adaptive Resonant Controller for GridConnected Converters in Distributed Power Generation Systems", in Proc. IEEE App. Power Elect. Conf. 2006, pp. 1601-1606

C. Meza, D. Jeltsema, J. Scherpen, D. Biel, "Passive PControl of a Grid-Connected Photovoltaic Inverter", in Proc. 17th IFAC World Congr., Seoul, Korea, pp. 55755580, July 6-11, 2008

S. Sanders and G. Verghese, "Lyapunov-based control for switched power converters", IEEE Trans. Power Elect., Vol. 7, No. 1, pp. 17-24, Jan. 1992,

C. Meza, D. Biel, D. Jeltsema, J. Scherpen, "LyapunovBased Control Scheme for Single-Phase Grid-Connected PV Central Inverters", IEEE Trans. Control Syst. Tech., Vol. 20, No. 2, pp. 520-529, March 2012,

H. El Fadil and F. Giri, "Climatic sensorless maximum power point tracking in PV generation systems", Control Engineering Practice, 19, pp. 513-521, 2011,

J. A. Gow and C. D. Manning, "Development of a photovoltaic array model for use in power-electronics simulation studies", in Proc. IEEE Elect. Power App., Vol. 146, No. 2, pp. 193-200, March 1999,

E. Saloux, A. Teyssedou and M. Sorin, "Explicit model of photovoltaic panels to determine voltages and currents at the maximum power point", Solar Energy, 85, pp. 713-722, 2011,

Aggarwal M., Kapoor A., Tripathi K.N., "Solar cell array parameters", Solar Energy Materials and Solar Cells, 45, pp. 377-384, 1997,

T. Bennett, A. Zilouchian, R. Messenger, "Photovoltaic model and converter topology considerations for MPPT purposes", Solar Energy, 86, pp. 2029-2040, 2012,

K. S. Narendra and A. M. Annaswamy, Stable Adaptive Systems, Prentice-Hall, 1989.

G. Kenne, T. Ahmed-Ali, F. Lamnabhi-Lagarrigue and A. Arzande, "Nonlinear systems time-varying parameter estimation: Application to induction motors", Electric Power Systems Research 78 (2008) pp. 1881-1888.

V. I. Utkin, Sliding Modes in Optimization and Control, Springer Verlag, 1992.

H. K. Khalil, Nonlinear Systems 2nd ed., PrenticeHall, 1996. 